\title{
CONTRIBUICÕES DO PASSADO EM AUXILIO AO FUTURO: TÉCNICAS DE RECUPERAÇÃO DE ÁREAS AFETADAS POR RUPTURA DE BARRAGEM DE REJEITO - UM ESTUDO DE CASO
}

\author{
Cássio Furtado Lima ${ }^{1}$, Rômulo Furtado Faria ${ }^{2}$, Raphael Magalhães Gomes Moreira ${ }^{3}$, \\ Ricardo da Silva Santos ${ }^{4}$, Sávio da Silva Berilli ${ }^{5}$ \\ ${ }^{1}$ Mestrando em Engenharia e Desenvolvimento Sustentável na Universidade Federal \\ do Espírito Santo (UFES), campus Vitória, Espírito Santo, Brasil. Prof. Instituto \\ Federal de Educação, Ciência e Tecnologia do Espírito Santo (IFES), Campus \\ Itapina, Espírito Santo, Brasil (cassio.lima@ifes.edu.br) \\ ${ }^{2}$ Prof. Mestre do Instituto Federal de Educação, Ciência e Tecnologia do Espírito \\ Santo (IFES), Campus Nova Venécia, Espírito Santo, Brasil \\ ${ }^{3}$ Prof. Doutor Instituto Federal de Educação, Ciência e Tecnologia do Espírito Santo \\ (IFES), Campus Itapina, Espírito Santo, Brasil \\ ${ }^{4}$ Prof. Mestre Instituto Federal de Educação, Ciência e Tecnologia do Espírito Santo \\ (IFES), Campus Itapina, Espírito Santo, Brasil \\ ${ }^{5}$ Prof. Doutor Instituto Federal de Educação, Ciência e Tecnologia do Espírito Santo \\ (IFES), Campus Itapina, Espírito Santo, Brasil
}

Recebido em: 08/04/2016 - Aprovado em: 30/05/2016 - Publicado em: 20/06/2016 DOI: 10.18677/Enciclopedia_Biosfera_2016_031

\begin{abstract}
RESUMO
A abordagem sobre procedimentos de recuperação e conservação edáfica é de suma importância, pois vários são os desafios que devem ser enfrentados visando o equilíbrio ambiental e o uso sustentável dos recursos naturais. Nesse contexto se torna imprescindível analisar as técnicas de mitigação de impactos utilizadas após a ruptura da barragem em Miraí (MG), em 2007. Considerado o segundo maior desastre ambiental de ruptura de barragem no Brasil, afetou a vida em vários ecossistemas naturais, somente superados pelo recente episódio ocorrido em Mariana (MG) em novembro de 2015, a análise de cenário é essencial para a elaboração e monitoramento de um plano eficaz de restauração da bacia hidrográfica do Rio Doce. Foram priorizadas ações de reconstituição do meio físico e biológico dos ecossistemas afetados, em cumprimento às exigências legais e ao devido respeito ao meio ambiente e a população das regiões afetadas. O presente trabalho buscou analisar as ações e medidas destinadas à recuperação ambiental da área diretamente afetada pela ruptura da barragem São Francisco, localizada na cidade de Miraí, MG e destinada à contenção dos rejeitos advindos do processo de beneficiamento da bauxita. Tudo isso com intuito de fornecer subsídios técnicos, aliados à busca de soluções para o maior acidente ambiental da história recente do Brasil, a contaminação do Rio Doce por meio de ruptura de barragem de rejeito. Especificamente, foram analisados os seguintes procedimentos: recuperação das calhas fluviais e dos diques temporários; estabilização dos taludes; recuperação das áreas marginais de proteção e o monitoramento hidrológico da região.
\end{abstract}

PALAVRAS-CHAVE: Conservacionista, edáfico, regulação. 


\title{
PAST CONTRIBUTIONS IN AID TO THE FUTURE: TECHNICAL AREAS AFFECTED BY RECOVERY REJECT DAM BREAK - A CASE STUDY
}

\begin{abstract}
The approach about procedures and edaphic conservation is utterly important, because there are many challanges that have to be faced considering the environmental balance and the use sustainable natural resources. In this context it is essential to analyze techniques of mitigation of impactes used after the dam break in Miraí (MG) in 2007. Considered the second largest environmental disaster of dam break in Brazil, it has affected the lives of many natural ecosystems, only overcome by the recent episode occurred in Mariana (MG) in November 2015, scenario analysis such analyses is essential for the development and monitoring of an effective restoration plan of Doce River's basin. Actions such as the rebuilding of the physical stock and the affected biological ecosystems were prioritized, in compliance with legal requirements and taking in consideration the environment and the population from the affected areas. This study aimed to analyze the actions and measures for the environmental recovery of the area directly affected by the rupture of the San Francisco dam, located in Miraí, MG and intended for containment of wastes arising from the bauxite beneficiation process. All this in order to provide technical inputs, coupled with the search for solutions to the biggest environmental accident of the recent history of Brazil, contamination of the Rio Doce through tailings dam break. Specifically, the following were analyzed: recovery of river runners and temporary dikes; stabilization of slopes; recovery of marginal areas of protection and hydrological monitoring in the region.
\end{abstract}

KEYWORDS: Conservation, edaphic, regulation.

\section{INTRODUÇÃO}

A exposição dos solos para práticas agrícolas, exploração agropecuária, mineração ou ocupações urbanas, em geral são acompanhadas de movimentação de terra e impermeabilização do solo. Estes usos abrem caminho para os processos erosivos e para o transporte de materiais orgânicos e inorgânicos, que são drenados até o depósito final nos leitos dos cursos d'água e dos lagos/lagoas. A degradação dos solos constitui um prejuízo socioeconômico e ambiental com graves riscos para as gerações futuras, uma vez que, envolve perda da biodiversidade e empobrecimento dos solos, os quais adquirem graus de vulnerabilidade relacionados às erosões, instabilidades hidrológicas, perda de matéria orgânica e mineral (PLEVIN et al., 2010).

Os empreendimentos de natureza diversa promovem a remoção do solo e deixam o substrato litólico exposto, com profundas modificações no equilíbrio ambiental dos ecossistemas (LAPOLA et al., 2010). As alterações são extensas e sujeitas a uma demanda de várias dezenas de anos, para que o local degradado adquira níveis de equilíbrio homeostático incipiente. Os substratos remanescentes tornam-se desprovidos de atributos físicos e químicos, que permitam a colonização vegetal espontânea, logo, tais áreas apresentam-se suscetíveis a ação dos processos erosivos (VALCARCEL \& D'ALTÉRIO, 1995).

Segundo REGENSBUGER (2004) os processos de degradação do solo são dinâmicos e responsáveis pela queda na qualidade. A degradação dos recursos naturais decorre de uso inadequado e, principalmente, da falta de planejamento ENCICLOPÉDIA BIOSFERA, Centro Científico Conhecer - Goiânia, v.13 n.23; p. 360 2016 
sobre o destino futuro da área. Mesmo com o acervo das técnicas conservacionistas, a degradação dos solos continua em franca expansão (SILVA et al., 2016). Segundo LIMA (2014) a proteção das áreas de recarga do lençol é essencial, pois além de aumentar os níveis de água no solo, contribui também, para a diminuição do assoreamento dos cursos hídricos, uma vez que a vegetação estabiliza o solo e diminui o carreamento de partículas.

FARIA (2010) ressalta a importância de analisar os procedimentos de recuperação da área afetada pela ruptura da barragem São Francisco, localizada no município de Miraí, MG em 2007, pois este foi na época, o maior acidente ambiental de extrusão de barragem no Brasil. Ressalte-se que, os impactos gerados se estenderam por aproximadamente $300 \mathrm{~km}$ até a bacia hidrográfica do Rio Muriaé. Em termos de proporção perde apenas, para o desastre ambiental gerado com a ruptura das barragens de Fundão e Santarém no munícipio de Mariana, MG ocorridos em 2015, que afetou drasticamente toda a fauna e flora da bacia hidrográfica do rio Doce.

A pesquisa em questão buscou analisar as ações e medidas destinadas à recuperação do solo, nas áreas afetadas pelo acidente, ressaltando técnicas e procedimentos fundamentais para estabilização dos taludes, recuperação das áreas marginais de proteção e o monitoramento hidrológico, tendo em vista as grandes alterações que as mesmas provocaram no ambiente.

\section{MATERIAL E MÉTODOS}

O estudo apresentado está ligado ao Instituto Federal do Espírito Santo, campus Itapina e Nova Venécia. Envolve uma pesquisa documental, na qual os dados coletados foram submetidos a uma análise de natureza qualitativa. A metodologia aplicada consiste em um estudo de caso, referente à ruptura do maciço da barragem São Francisco, no município de Miraí, MG, em janeiro de 2007 (Figura 1).

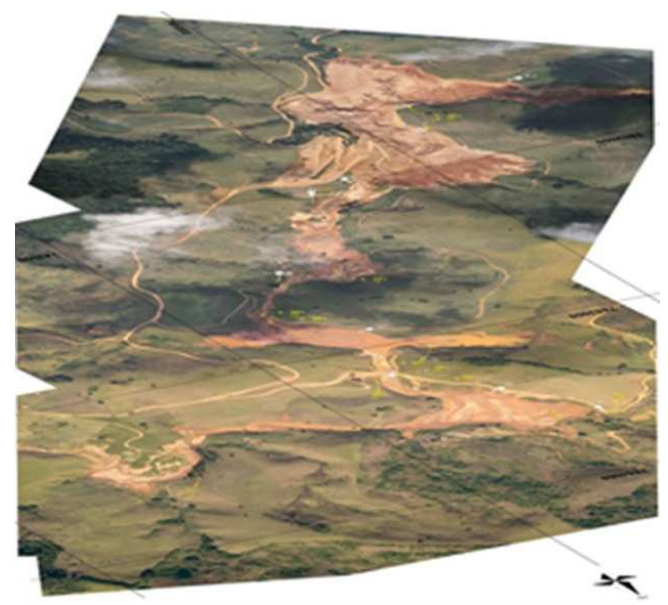

FIGURA 1: Foto aérea da barragem São Francisco após a ruptura em janeiro de 2007.

Fonte: PRAD (Plano de Recuperação de Área Degradada) da empresa.

O presente trabalho teve como foco realizar uma análise de cenário 2007/2015 entre o segundo maior desastre ambiental de ruptura de barragem no Brasil em Miraí (MG), e como este afetou a vida em vários ecossistemas naturais, ENCICLOPÉDIA BIOSFERA, Centro Científico Conhecer - Goiânia, v.13 n.23; p. 361 2016 
somente superados pelo recente episódio ocorrido em Mariana (MG) em novembro de 2015. Por meio das seguintes variáveis: recuperação das calhas fluviais, diques temporários, estabilização de taludes, recuperação das áreas marginais de proteção e o monitoramento hidrológico da região.

Visou também à busca por soluções mais eficazes que poderão ser reaplicadas no acidente ocorrido em novembro 2015, Mariana (MG). Além de retratar por meio de uma descrição detalhada, a multiplicidade dos aspectos presentes nos procedimentos de recuperação da área afetada pela ruptura da barragem de rejeitos. Por motivos éticos, inerentes à natureza da pesquisa, não foi divulgado o nome da empresa responsável.

\section{RESULTADOS E DISCUSSÃO}

A grande quantidade de "lama" (rejeito) lançada no rio Fubá, zona rural de Miraí, MG, chegou aos rios Muriaé e Paraíba do Sul, atingindo os municípios de Cardoso Moreira e Italva, além dos municípios à montante da bacia hidrográfica do Rio Muriaé (REIS et al., 2015). Segundo DUARTE (2008), nessa mesma barragem já havia observado anteriormente, em março de 2006, um vazamento de lama, que apesar de ter ocorrido em menor proporção, ocasionou danos ambientais, prejuízos materiais e suspensão no abastecimento de água em cidades de MG e RJ.

$\mathrm{O}$ acidente de janeiro de 2007 deixou mais de 500 pessoas desalojadas e 16.000 atingidas diretamente (REIS et al. 2015). A extensão dos danos e a apuração dos impactos certamente foram muito maiores do que o divulgado na época visto o quanto é difícil e complexo a avaliação e valoração do impacto ambiental em um curso hídrico. De acordo com (LIMA, 2014) a vazão do rio é o componente mais importante do ciclo hidrológico, uma vez que, exerce um efeito pronunciado sobre a sustentabilidade da vida, onde milhares de espécies da flora e fauna, inclusive a espécie humana, dependem a água que precisa ter a qualidade adequada para os diversos usos. Assim sendo, qualquer alteração ou degradação ambiental poderá comprometer grandes extensões deste ecossistema, durante vários anos. $O$ abastecimento de água na bacia do rio Muriaé nos estados de MG e RJ foi interrompido durante estes episódios por conta do comprometimento da qualidade da água (REIS et al., 2015). Fato semelhante ocorreu na bacia do rio Doce, onde várias cidades tiveram o abastecimento de água interrompido.

De acordo com HADEN et al. (2009), o intuito da gestão ambiental é a aplicação da inovação para alcançar a sustentabilidade, promovendo o desenvolvimento e aprendizagem contínua em acordo com os princípios da responsabilidade social, sem deixar de competir no mercado financeiro e de considerar os objetivos do negócio. Segundo PITELIS (2013) um empreendimento sustentável tem o dever de não somente garantir a viabilidade financeira, porém associar medidas mitigadoras dos danos aos recursos naturais e manter ou melhorar as condições de vida para a sociedade atual e futura. Com isso é imprescindível analisar os procedimentos na recuperação das áreas degradas pela ruptura da barragem, através das ações de proteção ambiental, estabilização edáfica, notadamente à física dos solos, além da reconstituição biológica nos ecossistemas afetados.

\section{Recuperação das Calhas Fluviais}

A desobstrução completa da calha fluvial do rio Fubá, pertencente à subbacia do rio Muriaé, que pertence à bacia hidrográfica do rio Paraíba do Sul, 
envolveu ações de contenção dos taludes das margens, gerados após a desobstrução. Em seguida, foram realizadas ações de recuperação da cobertura vegetal preexistente, com destinação adequada para o material removido da calha fluvial. FARIA (2010), ressalta que a desobstrução das calhas fluviais é de suma importância para o sucesso do plano de recuperação, pois constitui uma das etapas iniciais do processo. Portanto, se for mal executada comprometerá as demais atividades, de recuperação fauna do rio e flora da bacia.

As recuperações das calhas com a retirada do excesso de sedimentos permitiram que o rio retomasse o curso normal e evitou em épocas de intensa precipitação, que o nível do rio aumente atingindo a população ribeirinha. Segundo LIMA (2014) o coeficiente de escoamento superficial é uma variável importantíssima para o monitoramento hidrológico. Uma vez que o curso hídrico já está comprometido com uma grande quantidade de resíduos é fundamental diminuir o teor de partículas a serem carreadas, com a finalidade de mitigar o assoreamento do curso hídrico.

Ressalte-se que, a recuperação da calha do rio Fubá, segundo observações in loco, foi um procedimento que apresentou resultados satisfatórios. Considerando uma escala de maior impacto, esta técnica pode ser adaptada e utilizada na desobstrução do leito do rio Doce, para mitigação do impacto advindo da ruptura da barragem ocorrido em Mariana, MG em novembro de 2015.

Ao comparar o trabalho de FARIA (2010), que traz em sua essência, a descrição de uma abordagem técnica utilizada na recuperação de áreas afetadas por ruptura de barragens de rejeitos, com o panorama atual de degradação da bacia hidrográfica do rio Doce é de fundamental clareza, a observância da ausência de uma política adequada de gestão hídrica sustentável. Acredita-se que este fato se deve a um sucateamento dos órgãos ambientais fiscalizadores.

\section{Implantação de Diques Temporários}

As operações de recuperação ambiental foram desenvolvidas pela empresa na área do reservatório da barragem São Francisco, no restante dos terrenos ocupados pela unidade São Francisco (terrenos elevados), nas calhas fluviais e restauradas e/ou desassoreadas em todos os terrenos marginais. Destaca-se também, a própria remobilização natural dos sedimentos acumulados ao longo da área afetada. Diques filtrantes temporários foram implantados, diretamente sobre a calha fluvial, com o objetivo de retenção de sedimentos. Tais procedimentos minimizaram alguns impactos ambientais, relacionados principalmente com a qualidade das águas.

$\mathrm{Na}$ Figura 2, a seguir, mostra-se o dique de contenção de sedimentos implantado pela empresa no trecho compreendido entre a barragem São Francisco e a cidade de Miraí, local onde o potencial de aporte de sedimentos foi significativamente superior. 


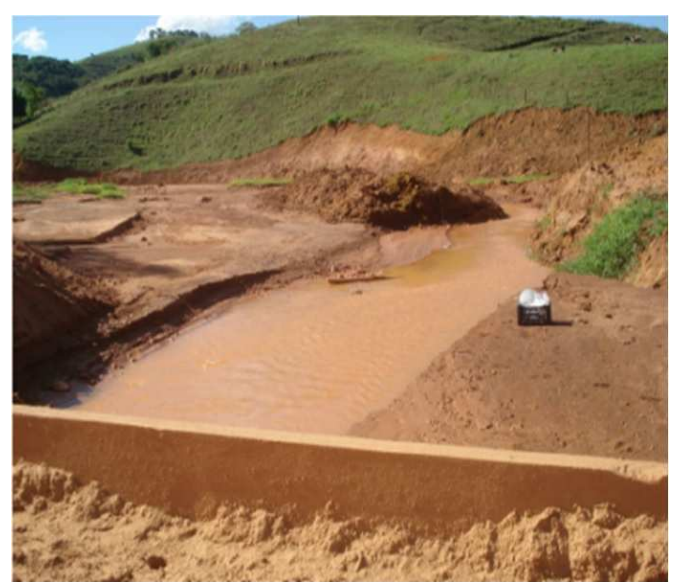

FIGURA 2: Dique que de contenção de sedimentos.

Fonte: PRAD da empresa.

Além da retirada de sedimentos os diques exercem uma função importantíssima, pois minimizam possíveis impactos ambientais devido ao represamento da água (QUEIROZ, et al., 2015). Confrontando casos distintos, mas de natureza similar, observa-se que até março de 2016 os rejeitos das barragens na cidade de Mariana, ainda escoam pela bacia hidrográfica, atingindo a calha principal do rio Doce, sem nenhuma ação para mitigação desse impacto, até o presente momento. Assim, a construção de diques temporários poderia ser uma técnica de mitigação a ser adotada.

\section{Estabilização de Taludes}

A estabilização de taludes foi executada ao término do diagnóstico ambiental e destinava-se ao trecho fluvial entre a confluência do rio Fubá, com o córrego Perobas e a cidade de Miraí. Neste trecho, constatou-se, de forma mais acentuada, a erosão e instabilidade dimensional de alguns taludes marginais, bem como, às porções de terreno que sofreram solapamento na borda do reservatório da barragem São Francisco. $O$ procedimento relacionado à estabilização dos taludes teve o intuído de auxiliar nas ações de monitoramento, na integridade das estruturas de drenagem e na contenção do maciço, durante o processo de recuperação das áreas diretamente afetadas (ADA). Para tal, foi elaborado um programa de avaliação geotécnica de estabilidade descrito no Quadro 1, a seguir.

QUADRO 1- Informações sobre o programa de avaliação geotécnica de estabilidade dos taludes recuperados.

\begin{tabular}{|c|c|c|c|}
\hline Programa & \multicolumn{3}{|c|}{ Avaliação Geotécnica de Estabilidade } \\
\hline Objetivo & $\begin{array}{l}\text { Monitorar a estabilidade das superfícies reconformadas dos } \\
\text { taludes que haviam sido instabilizados no trecho inicial da ADA, } \\
\text { bem como, a integridade das estruturas de drenagem e } \\
\text { contenção implantadas. }\end{array}$ \\
\hline $\begin{array}{c}\text { Local da } \\
\text { Avaliação }\end{array}$ & Parâmetros & Frequência & Período \\
\hline
\end{tabular}

ENCICLOPÉDIA BIOSFERA, Centro Científico Conhecer - Goiânia, v.13 n.23; p. 364 2016 


\begin{tabular}{|c|c|c|c|}
\hline $\begin{array}{l}\text { Taludes } \\
\text { marginais } \\
\text { recuperados no } \\
\text { trecho entre a } \\
\text { confluência do rio } \\
\text { Fubá com o } \\
\text { ribeirão Perobas } \\
\text { e a cidade de } \\
\text { Miraí. }\end{array}$ & $\begin{array}{l}\text { Movimentação do solo; } \\
\text { Presença de focos erosivos; } \\
\text { Presença de água; } \\
\text { Integridade das estruturas de } \\
\text { drenagem de crista e de } \\
\text { drenagem da área reconformada; } \\
\text { Integridade das estruturas de } \\
\text { contenção do maciço em } \\
\text { processo de estabilização. }\end{array}$ & Anual & $\begin{array}{l}\text { Iniciado } 6 \\
\text { meses após a } \\
\text { conclusão das } \\
\text { obras de } \\
\text { recuperação e } \\
\text { estabilização } \\
\text { dos taludes. }\end{array}$ \\
\hline
\end{tabular}

Fonte: PRAD da empresa

É importante ressaltar a notoriedade dos procedimentos adotados, nos moldes destacados no Quadro 1, com resultados bastante satisfatórios. Em determinadas áreas afetadas pela lama, o tratamento do solo consistiu na semeadura de mistura de sementes em substrato friável adubado, sem aplicação compulsória de cobertura morta, a fim de minimizar os gastos na aplicação do tratamento corretivo. Em outras áreas, cujas superfícies se mantiveram desnudas por tempo prolongado, expostas a ciclos de umedecimento e secagem e sujeitas ao impacto direto das chuvas, o solo foi preparado com o lançamento de cobertura vegetal morta, antes de receber as sementes. Concluiu-se que a eficácia destas ações foi maior, quanto mais imediata foi a aplicação nas áreas afetadas.

\section{RECUPERAÇÃO DOS TERRENOS MARGINAIS/LAGOAS}

$O$ desassoreamento e a consequente recuperação dos terrenos marginais e das lagoas ocorreram por meio da remoção dos sedimentos do interior destas estruturas e incorporados ao solo dos terrenos marginais vizinhos. Decorrido certo espaço de tempo, observou-se que a vegetação nativa das faixas marginais das lagoas haviam se recuperado, retomando as funções de proteção das margens contra a erosão, além de servir como filtro evitando que impurezas cheguem aos corpos d'água.

Acrescente-se também, a importante função ambiental de preservar o fluxo gênico de fauna e flora, uma vez que as APPs, principalmente aquelas das margens dos cursos d'água, são "corredores ecológicos" por excelência. O esforço de recuperar e manter preservada a vegetação nativa das faixas marginais das lagoas se está objetivamente, possibilitando a interligação destas com outros espaços territoriais especialmente protegidos, como no caso das áreas de Reserva Legal, Unidades de Conservação e outros remanescentes de vegetação nativa. Sugere-se, portanto, que este procedimento seja aplicado em outros trabalhos de recuperação de solos em desastres similares.

\section{PROGRAMA DE MONITORAMENTO HIDROLÓGICO}

Esse programa contemplou a qualidade dos recursos hídricos e fatores edáficos presentes na bacia, tais como: o escoamento superficial, a infiltração de água no solo e os níveis de água na percolação da área. Os ecossistemas 
aquáticos, complexos e dinâmicos, são influenciados por um conjunto de condições hidro biológicas, sendo que um dos mais importantes é o tempo de resiliência, que atua diretamente nos componentes físicos, químicos e biológicos da água. Tendo em vista a necessidade de utilizar a água da bacia hidrográfica do rio Muriaé foi realizada a análise de organismos planctônicos e bentônicos, a fim de determinar 0 estado trófico.

Considerando os ciclos biológicos mínimos para uma apreciação básica sobre a reversibilidade dos impactos nos ecossistemas aquáticos, afetados pela contaminação de rejeitos, o programa de monitoramento constou de um período amostral inicial de dois ciclos hidrológicos completos que correspondeu a dois anos. Ao término deste período, em virtude dos resultados se mostraram instáveis, o acompanhamento foi mantido por tempo indeterminado, a fim de buscar alcançar a retomada do equilíbrio. Também foi integrado a estas ações um programa de monitoramento da ictiofauna.

Corroborando com LIMA (2014) e CORRÊA (2014), a produção de água está associada à preservação e recuperação das nascentes e APP's. Neste sentido, tornou-se justificável e relevante a realização dessa etapa, já que o constante monitoramento do local associado ao processo de recuperação edáfica da bacia, possibilitou analisar a evolução da quantidade e qualidade da água. Percebeu-se também que fatores erosivos foram amenizados com o restabelecimento das APP's. De acordo com DADALTO (2014), recuperar e preservar as áreas marginais de cursos d'água significa garantir espaços, onde ecossistemas e habitats naturais são mantidos para abrigar a biodiversidade.

\section{CONCLUSÕES}

O rompimento de barragens de rejeitos na atividade de mineração pode trazer graves danos ao meio ambiente e à biodiversidade da região afetada. Dentre eles, pode-se citar o bloqueio de calhas fluviais, o assoreamento de terrenos baixos, várzeas e lagoas, as instabilidades edáficas que formam superfícies de possíveis deslizes, a presença de ravinas, a erosão e voçorocas, além da contaminação das águas de rios, lagoas e mares tornando-as impróprias para o consumo.

O monitoramento hidrológico, a limpeza de diques, a recuperação dos canais fluviais e terrenos marginais devem passar por uma constante vistoria, pois o processo de recuperação e a estabilização edáfica estão condicionados à extensão da área afetada e ao acompanhamento ao longo do tempo.

Observou-se que as técnicas de mitigação de impacto ambiental abordadas nesse estudo obtiveram resultados satisfatórios. Portanto, os procedimentos de monitoramento hidrológico, recuperação de matas ciliares e demais ecossistemas, estabilização de taludes, implantação de diques temporários e revitalização da calha do curso hídrico, se mostraram eficazes e podem ser recomendados em programas de proteção, recuperação e regulação de bacias hidrográficas.

Assim, sugere-se salvo as devidas proporções, a aplicação na recuperação de bacias hidrográficas afetadas pela ruptura de barragens de rejeitos da mineração, ou em outras situações de degradações semelhantes.

\section{REFERÊNCIAS}


CORRÊA, J. B. L. Processos Hidrológicos Quantitativos e Parametros de Qualidade da Água na Cultura de Macaúba (Acrocomia aculeata), Araponga MG. 49p. Tese (Doutorado) - Universidade Federal de Viçosa, Viçosa, Minas Gerais. 2014.

DADALTO, D. Proposta de Manejo em Mosaico Para Uma Bacia Hidrográfica de Uma Empresa do Setor Florestal em Felixlândia, Minas Gerais. Viçosa: Universidade Federal de Viçosa, 2014. 15p. Dissertação (Graduação) Universidade Federal de Viçosa, Viçosa, Minas Gerais. 2014.

DUARTE, A. P. Classificação das barragens de contenção de rejeitos de mineração e de resíduos industriais no estado de minas gerais em relação ao potencial de risco. 2008. Dissertação (Mestrado em Saneamento, Meio Ambiente e Recursos Hídricos) - Belo Horizonte, MG: UFMG.

FARIA, R. F. Análise dos Procedimentos de Recuperação das Áreas Afetadas pela Ruptura da Barragem São Francisco. Ouro Preto. Universidade Federal de Ouro Preto, 2010. 27p. Dissertação (Graduação) - Universidade Federal de Ouro Preto, Ouro Preto, Minas Gerais. 2010.

HADEN, S.S.P., OYLER, J.D., HUMPHREYS, J.H. Historical, practical, and theoretical perspectives on green management: an exploratory analysis. Jounal of Management Decision, Vol 47. 2009. Disponível em: <http://www.emeraldinsight. com/doi/abs/10.1108/00251740910978287>. Doi: 10.1108/00251740910978287.

LAPOLA, D. M.; SCHALDACH, R.; ALCAMO, J.; BONDEAU, A.; KOCH, J.; KOELKING, C.; PRIESS, J. A. Indirect land-use changes can overcome carbon savings from biofuels in Brazil. Journal Proceedings of the National Academy of Sciences of the United States of America. v. 07, n. 08, p.3388-3393. Arizona, Estados Unidos. Janeiro, 2010. Disponível em: <http://www.pnas.org/ content/107/8/3388.full.pdf $>$. Doi:10.1073/pnas.0907318107.

LIMA, C. F. Monitoramento Hidrológico da Microbacia do Janjão, com Platio da Acronomia aculeata, no Município de Viçosa, MG. Viçosa: Universidade Federal de Viçosa, 2014. 15p. Dissertação (Graduação) - Universidade Federal de Viçosa, Viçosa, Minas Gerais. 2014.

PITELIS, C. N. Towards a More 'Ethically Correct' Governance for Economic Sustainability. Journal of Business Ethics. Cambridge, Reino Unido. Janeiro, 2013. Disponível em: <https://www.sussex.ac.uk/webteam/gateway/file.php?name=pitelisseminarpaper.pdf\&site=25>. Doi: 10.1007/s10551-012-1616-8

PLEVIN, R. J.; O'HARE, M.; JONES, A.D.; TORN, M. S.; GIBBS, H. K. Greenhouse gas emissions from biofuels' indirect land use change are uncertain but may be much greater than previously estimated. Journal Environmental Science \& Technology, v.44, n.21, p.8015-8021. Outubro, 2010. Disponível em: <http://pubs.acs.org/doi/pdf/10.1021/es101946t>. Doi:10.1021/es101946t. 
QUEIROZ, R. Diagnóstico de impactos em área de instalação de pequena central hidrelétrica no município de Taquaruçu do Sul/RS. Revista Eletrônica em Gestão, Educação e Tecnologia Ambiental - REGET, V. 19, 1, p.96-104. Disponível em: $<$ http://cascavel.cpd.ufsm.br/revistas/ojs2.2.2/index.php/reget/article/view/15538/ pdf>. Acesso em: 08104l2016. Doi: 10.5902/22361170.

REGENSBURGER, B. Recuperação de áreas degradadas pela mineração de argila através da regularização topográfica, da adição de insumos e serapilheira, e de atratatores da fauna. 2004. 15-35p. Dissertação (Mestrado)Universidade Federal de Santa Catarina, Florianópolis, SC.

REIS, C. H.; AMORIM, R. R.; FERREIRA, C. Caracterização do Uso e Ocupação das Terras de Áreas com Risco à Inundação em Cardoso Moreira e Italva, Rio De Janeiro. Revista Caminhos da Geografia. v. 16, n. 55, p. 01-17. Setembro, 2015. Uberlândia, Minas Gerais. Disponível em: <http://www.seer.ufu.br/index.php/ caminhosdegeografia/article/view/29169/17251> Acesso em: 08104/2016 DOI: Não Informado.

SILVA, L. F.; CAVALCANTE, C. P.; SILVA, A. G. da. Evaluation of degradation of environmental resources: A case study on a rural property Pilõezinhos-PB. Revista Monografias Ambientais - REMOA. v. 15, n.1, jan-abr. 2016, p.132-140. Santa Maria, RS. Disponível em: <http://cascavel.ufsm.br/revistas/ojs-2.2.2/ index.php/remoa/article/view/19965/pdf> Doi: 10.5902/22361308.

VALCARCEL, R \& D'ALTÉRIO. Medidas físico-biológicas de recuperação de áreas degradadas: almofadas. Relatório final do CNPq, impresso, Seropédica: UFRRJ, 1995. 